\title{
Early-life stress impairs postnatal oligodendrogenesis and adult emotional behaviour through activity-dependent mechanisms
}

\author{
Anne Teissier ${ }^{1,2,7} \cdot$ Corentin Le Magueresse $^{1,2} \cdot$ Jimmy Olusakin $^{1,2} \cdot$ Belmira L. S. Andrade da Costa ${ }^{3}$. \\ Angela M. De Stasi ${ }^{2,4} \cdot$ Alberto Bacci $^{2,4}$ - Yuka Imamura Kawasawa ${ }^{5} \cdot$ Vidita A. Vaidya $^{6} \cdot$ Patricia Gaspar $\mathbb{D}^{1,2,4}$
}

Received: 24 August 2018 / Revised: 27 June 2019 / Accepted: 9 July 2019 / Published online: 22 August 2019

(c) The Author(s) 2019. This article is published with open access

\begin{abstract}
Exposure to stress during early life (infancy/childhood) has long-term effects on the structure and function of the prefrontal cortex (PFC), and increases the risk for adult depression and anxiety disorders. However, little is known about the molecular and cellular mechanisms of these effects. Here, we focused on changes induced by chronic maternal separation during the first 2 weeks of postnatal life. Unbiased mRNA expression profiling in the medial PFC (mPFC) of maternally separated (MS) pups identified an increased expression of myelin-related genes and a decreased expression of immediate early genes. Oligodendrocyte lineage markers and birthdating experiments indicated a precocious oligodendrocyte differentiation in the mPFC at P15, leading to a depletion of the oligodendrocyte progenitor pool in MS adults. We tested the role of neuronal activity in oligodendrogenesis, using designed receptors exclusively activated by designed drugs (DREADDs) techniques. $\mathrm{hM} 4 \mathrm{Di}$ or hM3Dq constructs were transfected into mPFC neurons using fast-acting AAV8 viruses. Reduction of mPFC neuron excitability during the first 2 postnatal weeks caused a premature differentiation of oligodendrocytes similar to the MS pups, while chemogenetic activation normalised it in the MS animals. Bidirectional manipulation of neuron excitability in the mPFC during the P2-P14 period had long lasting effects on adult emotional behaviours and on temporal object recognition: hM4Di mimicked MS effects, while hM3Dq prevented the pro-depressive effects and short-term memory impairment of MS. Thus, our results identify neuronal activity as a critical target of early-life stress and demonstrate its function in controlling both postnatal oligodendrogenesis and adult mPFC-related behaviours.
\end{abstract}

\section{Introduction}

The environment a child encounters during early life plays a critical role in normal brain development. Thus, early-life stress (ELS), which includes various forms of child abuse

Supplementary information The online version of this article (https:// doi.org/10.1038/s41380-019-0493-2) contains supplementary material, which is available to authorised users.

Anne Teissier

anne.teissier@inserm.fr

$\triangle$ Patricia Gaspar

Patricia.gaspar@inserm.fr

1 INSERM, Institut du Fer à Moulin, UMR-S 1270, Sorbonne Université, Paris, France

2 Sorbonne Université, Paris, France

3 Physiology and Pharmacology Department, Federal University of Pernambuco, Recife, Brazil and neglect, has repeatedly been shown to increase the risk to develop psychiatric disorders [1-4]. Current evidence that ELS alters developmental trajectories is essentially based on brain imaging studies in adult patients who experienced childhood adversity. These studies showed altered brain activity and myelin content, notably affecting the medial prefrontal cortex (mPFC), which constitutes a critical hub for emotional control and cognition [5-8]. Since the mPFC has a protracted postnatal development, it

4 Institut du Cerveau et de la Moelle épinière, CNRS UMR 7225Inserm U1127, Paris, France

5 Departments of Pharmacology and Biochemistry and Molecular Biology, Institute for Personalized Medicine, Penn State University College of Medicine, Hershey, PA, USA

6 Department of Biological Sciences, Tata Institute of Fundamental Research, Mumbai 400005, India

7 Present address: Institute of Psychiatry and Neuroscience of Paris, INSERM U1266, Université de Paris, Paris, France 
is a likely target for environmental challenges occurring in childhood.

To identify the pathophysiological mechanisms leading to ELS phenotypes, in particular as regards mood and anxiety disorders, several preclinical models have been developed [9, 10]. The most common involves daily maternal separation of rodent pups during the early postnatal period, a time when pups normally spend most of their time huddled in the mother's nest [9, 11]. Maternal separation (MS) during the first postnatal weeks induces a permanent impairment of emotional and cognitive behaviours, together with an altered physiology and myelination of the mPFC [12-15]. Strikingly, these behavioural and structural defects closely reproduce the phenotypes of humans exposed to childhood trauma $[10,16]$. Several of these alterations are already apparent at juvenile or adolescent stages [14, 17-20] but the causal mechanisms remain poorly understood.

Here, we focused on cellular and molecular changes occurring in the mPFC at the outset of ELS. We started with an unbiased transcriptomic screen of the mPFC, comparing MS with standard facility raised (SFR) pups. We identified major changes in gene pathways associated with myelination and neuronal activity. Turning to cellular analyses, we document a precocious differentiation of oligodendrocyte progenitor cells (OPC) in P15 MS pups, which coincides with an increase in myelin-related transcripts, and a depletion of the OPC pool in adults. Considering the important role of neuronal activity in adult myelination [21], we tested the contribution of altered $\mathrm{mPFC}$ neuronal activity during development on maternal separation phenotypes using designed receptors exclusively activated by designed drugs (DREADDs) [22]. We find that local and transient decrease in neuronal activity from $\mathrm{P} 2$ to $\mathrm{P} 14$ is sufficient to induce premature OPC differentiation, while transient increase in neuronal activity in MS pups, prevents it. Additionally, we demonstrate that some of the behavioural symptoms induced by ELS in adulthood are modulated by changes in mPFC neuronal activity during development.

\section{Material And methods}

\section{Animals}

All experiments performed in mice were in compliance with the standard ethical guidelines (European Community Guidelines and French Agriculture and Forestry Ministry Guidelines for Handling Animals-decree 87849). All procedures have been approved by the ethical commity of the region Ile de France (Comité Darwin, agreement 09047.04). All dams were first-time pregnant $\mathrm{BALB} / \mathrm{cJ} \mathrm{Rj}$ mothers shipped from Janvier Labs $\sim 7$ days before delivery. At arrival, pregnant females were group-housed by two and litter sizes were homogenised from 8 to 14 animals with both genders represented. After weaning at $\mathrm{P} 21$, all the mice were grouphoused (4-5 per cage), and kept under standard laboratory conditions $\left(22 \pm 1{ }^{\circ} \mathrm{C}, 60 \%\right.$ relative humidity, $12-12 \mathrm{~h}$ light-dark cycle, food and water ad libitum) in ventilated racks. Males and females were similarly exposed to maternal separation protocol (MS pups) and/or viral injections, but only males were used for the experiments while females were sacrificed at weaning except for: (i) a first cohort of MS animals in which both genders were behaviourally tested and (ii) females receiving viral injection at $\mathrm{P} 1$ and sacrificed at $\mathrm{P} 2$, P4, P6, P8 and P10 for time course of viral expression or at $\mathrm{P} 8$ or P14 to test for CNO efficacy using Egr1 staining.

\section{Maternal separation protocol}

Maternal separation of pups from their dams was conducted daily between 1.00 and 4.00 P.M., starting at postnatal day 2 (P2) and terminating at P14. Briefly, dams were first removed from the home cage and placed into a clean cage, then pups, were collected and placed together into another clean cage. The two cages were separated at some distance to avoid vocalised communications. After $180 \mathrm{~min}$, pups and then dams were returned to their home cages. Control animals were standard facility-reared (SFR) and did not receive extra handling. Animals were sexed at P1 to allow random but homogeneous distribution of males, a first cage change occurred at P2 and then every week.

\section{RNA-sequencing}

For the transcriptomic profiling, bilateral mPFCs from SFR $(n=12)$ and MS $(n=12)$ animals were dissected manually at P15 (Fig. 1a), fast frozen on dry ice and collected in $100 \mu \mathrm{l}$ of RNALater ${ }^{\circledast}$ (Ambion, Life Technology). Three unilateral mPFCs from different litters were pooled to generate four samples per group. Samples were shipped to Penn State Hershey Genome Sciences Facility (USA). Total RNA was extracted using mirVana kit (Thermo Fisher Scientific) and a bead mill homogeniser (Bullet Blender, Next Advance) together with an equivalent mass of stainless steel beads (Next Advance) were used to homogenise the tissue. The complementary DNA (cDNA) libraries were prepared using SureSelect Strand-Specific RNA Library Preparation Kit (Agilent Technologies) and RNA and cDNA qualities were assessed using BioAnalyzer Kits (Agilent Technologies, RNA 6000 Nano and High Sensitivity DNA). The 12 libraries were loaded onto TruSeq SR v3 flow cells on an Illumina HiSeq 2500 and run for 50 cycles using a single-read recipe (TruSeq SBS Kit v3). The Illumina CASAVA pipeline Version 1.8 was used to extract de-multiplexed sequencing reads and FastQC 
a

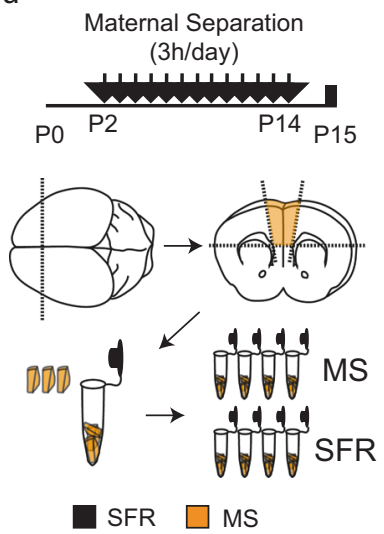

b

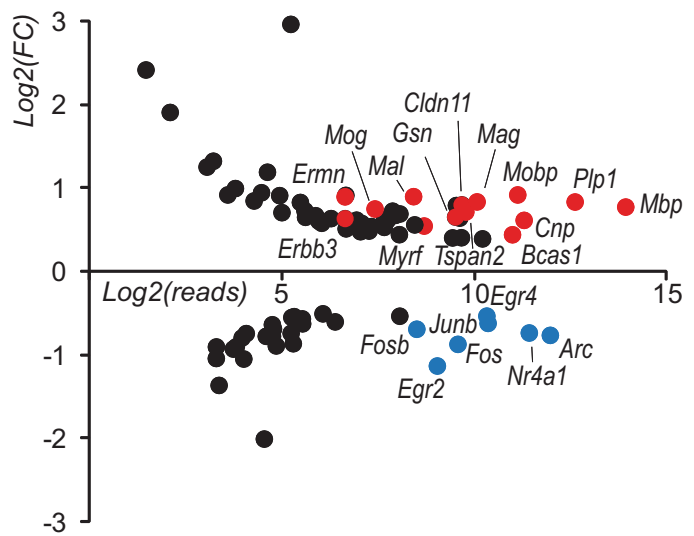

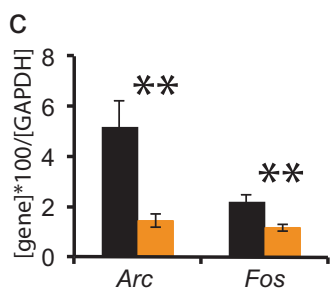

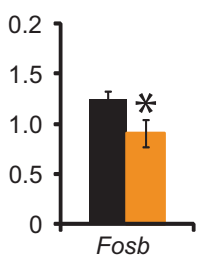

Fig. 1 Analysis of differentially expressed genes in the medial prefrontal cortex (mPFC) of P15 animals exposed to maternal separation highlights myelin ensheathment and immediate early genes. a Schematic presentation of the experimental paradigm of the maternal separation (MS pups) versus standard facility (SFR pups) protocols and the dissection of $\mathrm{mPFC}$ at P15 to generate four samples per condition, each including three unilateral mPFC. b Graph shows with a $\log 2$ scale the fold change (FC) of expression induced by maternal

(version 0.11.2) was used to validate the quality of the raw sequence data. Additional quality filtering was performed using FASTX-Toolkit with a quality score cutoff of 20 . Next, alignment of the filtered reads to the mouse reference genome $(\mathrm{mm} 10 \mathrm{~mm} 10)$ was done using Tophat (version 2.0.9) allowing two mismatches. Raw read counts were calculated using HTSeq-count [23]. The "remove unwanted variation" (RUVg) strategy ("RUVseq" R package, v3.1) as described by Risso et al. [24] was used along with edgeR [25] to identify differentially expressed genes (DEG) between MS and SFR animals. First, we normalised the raw read counts by selecting a set of "in-silico empirical" negative controls, i.e., 5000 least significantly DEG genes based on a first-pass DEG analysis performed prior to normalisation. Normalised read counts were applied, using the negative binomial GLM approach implemented in edgeR, using the Likelihood Ratio Test method. Significantly DEG were defined to be those with $q$-value $<0.05$ calculated by the Storey et al. method [26].

\section{Reverse transcription quantitative PCR (RT-qPCR)}

mPFCs were dissected and homogenised in $1 \mathrm{ml}$ of TriReagent (Sigma-Aldrich) using a Polytron ${ }^{\circledR}$ PT13000D

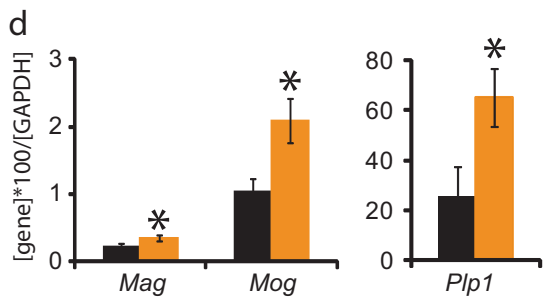

separation according to the number of reads detected in control samples for the genes with $q<0.05$ and FC $>1.3$ or FC $<0.7$. Myelinensheathment genes are highlighted in red, and immediate early genes in blue. c, d Graphs show the quantitative PCR (qPCR) results obtained in a second experimental cohort to determine the expression levels of IEG (C, $n=7$ SFR animals and $n=6 \mathrm{MS}$ ) and genes related to myelin ensheathment (D, $n=6$ SFR animals and 9 MS). ${ }^{*} p<0.05$, $* * p<0.01$

(Kinematica) and RNA was extracted according to standard protocols. Genomic DNA was removed by digestion with Amplification Grade DNase I (Sigma-Aldrich). First-strand cDNA was synthesised by reverse transcription of $5 \mu \mathrm{g}$ of total RNA with Superscript-II reverse transcriptase (Invitrogen) according to standard protocols. Reverse transcriptase was omitted in some samples as negative controls. Relative expression levels of Gapdh, Arc, Fos, Fosb, Mag, Mog and Plpl mRNAs were determined by real-time quantitative PCR (RT-qPCR) using Absolute SYBR Green Mix (ABgene) and sets of specific primers (Supplementary Table 2) on a 96-wells plate (Thermo Scientific AB-0600). Gene expression was normalised to mouse Gapdh mRNA expression. Data were analysed with the $2^{-\Delta \mathrm{Ct}}$ method on MxPro qPCR software (Agilent Technologies), and values are expressed as the mean of duplicates.

\section{AAV injections in the PFC}

AAV8-hSyn-hM3D(Gq)-mCherry, AAV8-hSyn-hM4D (Gi)-mCherry, AAV9-hSyn-hChR2(H134R)-EYFP or AAV8-hSyn-EGFP viruses (Addgene \#50474, \#50475, \#26973 or \#50465) were used as specified. The titres were within the range of $10^{12}-10^{13}$ particles $/ \mathrm{ml}$. Injections were 
done on P1 mice pups anaesthetised on ice (1 min or until the absence of pinch reaction) and maintained on a frozen pad during all the procedure ( $\max 8 \mathrm{~min}$ ). At the beginning of the experiment, litters were separated from the dams and placed into a heated cage with bedding. Pups were then individually anaesthetised and maintained in a prone position with the skull flat, the skin was gently opened as a single incision with a razor blade. The mPFC was targeted using as a reference the inferior cerebral vein $(\sim 2-3 \mathrm{~mm}$ posteriorly) and the superior sagittal sinus $(\sim 2 \mathrm{~mm}$ laterally). Bilateral injections $(200 \mathrm{nl}$ each) were performed $1.1 \mathrm{~mm}$ deep from the skull surface using a pulled glass capillary $(30-50 \mu \mathrm{m}$ tip diameter PCR micropipette, Drummond Scientific Company) mounted on a hydraulic micromanipulator MO-10 (Narashige, Japan). After the injection, the pups were immediately removed from the ice, the opened skin was sealed with tissue glue (Vetbond \#1469, 3M, France) and pups were gently warmed up. All pups from the same litter were injected to avoid competition for feeding and were returned together to their home cage at the end of the procedure. Careful supervision was provided during the hours following the procedure to ensure mother acceptance and re-glue the scars if necessary. Afterwards, cages were returned to the normal facility and daily supervision was provided.

\section{Chronic CNO injections}

A saline solution (SAL, $0.9 \% \mathrm{NaCl}$ ) or $5 \mathrm{mg} / \mathrm{kg} \mathrm{CNO}+$ $0.9 \% \mathrm{NaCl}$ solution was injected daily from $\mathrm{P} 2$ to $\mathrm{P} 14$, either subcutaneously (P2-P4) or i.p. (P5 onward). For SFR + hM4Di and SFR + GFP cohorts, injections were done at 1 p.m. For the MS + hM4Dq cohort, injections were done at 4 p.m. at the end of the maternal separation period.

\section{Tissue processing}

Mice were sacrificed at different postnatal times (P2-P15) and adult stages. All mice were anaesthetised (Pentobarbital $0.5 \mathrm{mg} / \mathrm{g}$ ) and fixed by intracardiac perfusion of $4 \%$ paraformaldehyde in $0.1 \mathrm{M}$ phosphate-buffer $(\mathrm{PB} \mathrm{pH}=7.2$ ) using a peristaltic pump (Thermoscientific Masterflex). Brains were quickly removed, post-fixed overnight in the same fixative solution, and cryoprotected 2 days in $30 \%$ sucrose containing $0.01 \%$ sodium azide. Thirty-five micrometre thick coronal sections were prepared using a cryostat (Leica) or a cryotome (Microm Microtech). Serial sections from the whole brain were collected as series of 6 . Tissue sections were used immediately for immunohistochemistry or stored at $-20{ }^{\circ} \mathrm{C}$ in a cryoprotective solution (30\% ethylene glycol and $30 \%$ sucrose in $0.1 \mathrm{M}$ phosphate buffer, $\mathrm{pH}=7.4$ ).

\section{Immunohistochemistry}

Immunolabelling techniques were used on free-floating sections, and all the washes and antibody incubations were performed in blocking solution containing $1 \%$ horse serum and $0.2 \%$ triton in phosphate buffer saline (PBST). The following primary antibodies were applied for $24 \mathrm{~h}$ (or $48 \mathrm{~h}$ for anti-cFos antibodies) at $4{ }^{\circ} \mathrm{C}$ : mouse anti-APC/CC1 Ab7 (Millipore, OP80, 1:100), chicken anti-mCherry (Abcam, 205402, 1:1000), rat anti-Ctip2 monoclonal (Abcam, ab18465, 1:500), rabbit anti-cFos (Synaptic System 226003, 1:1000) for P9 MS/SFR pups, rabbit anti-cFos antiserum (Abcam, 190289, 1:1000) for DREADDs experiments, mouse anti-NeuN (US Biological, 1:600), rabbit anti-Olig2 (Millipore Ab9610, 1:1000), rat antiPDGFR $\alpha$ (BD Bioscience, 558774, 1:600) and rabbit antiTbr1 (Abcam, 1:1000). The corresponding fluorescent donkey antisera secondary (Jackson ImmunoResearch, 1:200) were applied for $2 \mathrm{~h}$ at room temperature. Sections were subsequently mounted using the SlowFade (Molecular Probes 536939).

\section{Image acquisition}

Fluorescent images were acquired on a Leica SP5 confocal system equipped with an Argon laser (for the $488 \mathrm{~nm}$ excitation), a Diode $561 \mathrm{~nm}$ and $\mathrm{HeNe} 633 \mathrm{~nm}$. Z-series stacks of confocal images were acquired at $1024 \times 1024$ pixels resolution, with a pinhole set to one Airy unit and optimal settings for gain and offset.

\section{Western blot}

P15 SFR and MS mice were decapitated and MPFC were macroscopically dissected on ice. Tissue from two brains were pooled and $600 \mu \mathrm{l}$ of lysis buffer [Tris $100 \mathrm{mM}$ pH 7.6, EDTA $0.5 \mathrm{M} \mathrm{pH} \mathrm{8,1 \%} \mathrm{Triton} \mathrm{X-100} \mathrm{and} \mathrm{protease}$ inhibitor cocktail (\#P8340, Sigma, France)] was added to the tissue and sonicated on ice for $5 \mathrm{~min}$. Samples were centrifuged at $13,000 \times g\left(30 \mathrm{~min}\right.$ at $\left.4{ }^{\circ} \mathrm{C}\right)$, supernatants were collected, and an aliquot from each sample was used for protein determination (bicinchoninic assay). A total of $40 \mu \mathrm{g}$ of each protein extract sample was subjected to sodium dodecyl sulfate polyacrylamide gel electrophoresis, using NuPage 4-12\% Bis-Tris polyacrylamide gels (Invitrogen, France). Proteins were blotted onto a $0.45-\mu \mathrm{m}$ nitrocellulose membrane (Thermo Fisher, France). Membranes were blocked $(1 \mathrm{~h})$ in $5 \%$ non-fat milk dissolved in PBS $(\mathrm{pH}=7.6)+0.1 \%$ Tween (PBST) and incubated overnight in 1:1000 rabbit anti-PLP1 (Abcam, Cat. \# ab28486) or 1:1000 mouse anti-Tuj1 (BioLegend, Cat. \# $801201)$ at $4{ }^{\circ} \mathrm{C}$. After three rinses in PBST, membranes 
were incubated in Alexa Fluor 790-conjugated donkey anti-rabbit secondary antiserum (1:10,000, Jackson ImmunoResearch, \#711-655-152) or Alexa Fluor 680conjugated donkey anti-mouse secondary antiserum (1:20,000, Jackson ImmunoResearch, \#715-625-151) at room temperature for $90 \mathrm{~min}$. Data were expressed as the ratio between the bands intensity of PLP1 and Tuj1.

\section{Acute slice preparation and electrophysiology}

P9-P11 mice were sacrificed by decapitation and the brains were immediately removed from the skull and placed in oxygenated $4{ }^{\circ} \mathrm{C}$ artificial cerebrospinal fluid (ACSF1), with the following concentrations in mM: $125 \mathrm{NaCl}, 2.5 \mathrm{KCl}, 25$ glucose, $25 \mathrm{NaHCO}_{3}, 1.25 \mathrm{NaH}_{2} \mathrm{PO}_{4}, 2 \mathrm{CaCl}_{2}$ and $1 \mathrm{MgCl}_{2}$, and continuously bubbled with $95 \% \mathrm{O}_{2}-5 \% \quad \mathrm{CO}_{2}$. Slices $(250 \mu \mathrm{m})$ were cut using a vibroslicer (HM650V, Microm) and incubated at $32{ }^{\circ} \mathrm{C}$ for $20 \mathrm{~min}$ and then at room temperature $\left(20-25^{\circ} \mathrm{C}\right)$. For patch-clamp recordings, slices were transferred to the recording chamber and continuously superfused with ACSF2 $\left(30-32^{\circ} \mathrm{C}\right)$, with the following concentrations in mM: $125 \mathrm{NaCl}, 3.5 \mathrm{KCl}, 25$ glucose, 25 $\mathrm{NaHCO}_{3}, 1.25 \mathrm{NaH}_{2} \mathrm{PO}_{4}, 1 \mathrm{CaCl}_{2}$ and $0.5 \mathrm{MgCl}_{2}, \mathrm{pH} 7.2$, and continuously bubbled with $95 \% \mathrm{O}_{2}-5 \% \mathrm{CO}_{2}$. mCherry ${ }^{+}$ neurons were visually identified using an upright microscope (BX 51, Olympus) equipped with standard epifluorescence. To minimise cell-to-cell variability, we selectively recorded from thick-tufted layer five pyramidal neurons, which were identified based on their pyramid-like soma and stereotypical dendritic morphology with a prominent apical dendrite. Patch-clamp pipettes (4-6 Mohm resistance) were prepared from borosilicate glass (BF15086-10; Harvard Apparatus) with a DMZ pipette puller (Zeitz). Patch-clamp experiments were performed using the following intracellular solution: $105 \mathrm{~K}$-gluconate, 10 HEPES, 10 phosphocreatine-Na, 4 MgATP, $30 \mathrm{KCl}(\mathrm{pH}$ 7.25, adjusted with $\mathrm{KOH}) . \mathrm{GABA}_{\mathrm{A}}$ receptors were blocked with $10 \mu \mathrm{m}$ SR95531 hydrobromide (Gabazine, HelloBio), AMPA receptors with $10 \mu \mathrm{M}$ 6-cyano-7-nitroquinoxaline2,3-dione (CNQX, Hello Bio Inc), and NMDA receptors with $50 \mu \mathrm{M}$ d-APV (HelloBio). To activate DREADDs in current-clamp experiments, mCherry $^{+}$thick-tufted layer five pyramidal neurons were patch-clamped in the presence of CNO $(30 \mu \mathrm{M})$ or clozapine $(1 \mu \mathrm{M})$ in the ACSF. Intrinsic excitability was measured as the firing frequency in response to incremental depolarising current steps. To measure CNO-induced currents in voltage-clamp experiments, holding current was recorded every $2 \mathrm{~s}$. Thirty consecutive sweeps starting 2 min after CNO application were averaged and compared to the average of 30 sweeps immediately prior to CNO application ("baseline"). Barium chloride (100 $\mu \mathrm{M}$, Sigma) was always applied in the ACSF at the end of the experiment to block CNO-induced currents. The junction potential $(-5 \mathrm{mV})$ was not corrected for. Data acquisition was performed using Patchmaster software (Heka Elektronik). Signals were sampled at $20 \mathrm{kHz}$ and filtered at $3 \mathrm{kHz}$, and off-line analysis was performed using Igor (WaveMetrics Inc.). The experimenter was blind to both treatment and/or viral subtype.

\section{Behavioural studies}

Female and male mice were tested separately for emotional behaviours starting at P80 and animals were generated to reach at least ten per group. The tests were performed in the following order: open field (OF), elevated plus maze (EPM), marble burying, sequential object recognition (SNOR), splash test and the 2-days forced swim test, with 3 to 7 days between each test according to the stressful character of the test. All behavioural testing was done during the light cycle between 9.00 a.m. and 6.00 p.m. Animals were habituated to the testing room for at least $30 \mathrm{~min}$ and the experimenter was blind to animal treatment . To eliminate odour cues, each apparatus was thoroughly cleaned with alcohol after each animal. Testing took place under dim light conditions (40 lux) for OF, EPM, SNOR and splash test to enhance tracking of white mice, and under normal light conditions (80 lux) for the other tests.

\section{Open field}

Mice were placed in the centre of rectangular plastic grey boxes (H24.5 x L35 x L55 cm) and activity was recorded for $20 \mathrm{~min}$. Total distance and time spent in the centre of the $\mathrm{OF}$ were automatically recorded using AnyMaze tracking software (Stoelting Co).

\section{Elevated plus maze}

The maze is a grey plus-crossed-shaped apparatus, with two open arms $(30 \mathrm{~cm})$ and two closed arms $(30 \mathrm{~m})$ of $5 \mathrm{~cm}$ width, linked by a central platform and located $40 \mathrm{~cm}$ above the floor. Mice were individually put in the centre of the maze facing an open arm and were allowed to explore the maze for $10 \mathrm{~min}$. The time spent and the entries into the open arms were automatically measured using AnyMaze tracking software and used as anxiety indexes.

\section{Marble burying}

Housing cages $(20 \times 36 \mathrm{~cm})$ were filled with $6 \mathrm{~cm}$ of clean wood bedding with 12 marbles uniformly spaced [27]. Mice 
were individually placed in a corner of the cage and the number of marbles buried on more than $2 / 3$ of their surface was manually recorded every minute for $20 \mathrm{~min}$.

\section{Splash test}

Group housed animals were placed in a new cage with bedding for at least $30 \mathrm{~min}$. Mice were then individually sprayed on the back twice $(\sim 2 \times 0.6 \mathrm{ml})$ with $20 \%$ sucrose solution in water and placed in a corner of their home cage. Grooming behaviours, including licking, stroking and scratching were manually recorded using AnyMaze software keys.

\section{Sequential novel object recognition}

This is an adapted version of the novel object recognition test considered to be more specific to $\mathrm{mPFC}$ functioning [28]. Animals were first habituated to the arena (same grey boxes as $\mathrm{OF}$ ), without stimuli for 10 min daily for 2 days before the behavioural testing. The task comprises two sample phases and one test trial. In each sample phase, mice were allowed to explore two copies of an identical object for a total of $4 \mathrm{~min}$. Different objects were used for sample phases 1 and 2 (miniature car then plastic hook, or glass tube then cork of Falcon tube, or plastic mug then cell culture flask), with a $1 \mathrm{~h}$ delay between the sample phases. The test trial ( $3 \mathrm{~min}$ ) was given $3 \mathrm{~h}$ after sample phase 2 and consisted in placing together one copy of each set of objects. If temporal order memory is intact, the subjects will spend more time exploring the less recent object (from sample 1) compared with the more recent object (from sample 2). The positions of the two objects were counterbalanced between animals.

\section{Porsolt forced swim test}

Testing of behavioural despair was carried out on 2 consecutive days using a glass cylinder $(40 \times 20 \mathrm{~cm}$ diameter $)$ filled with water $\left(23-25^{\circ} \mathrm{C}\right)$ up to $3 / 4$. Mice were tested once a day for $6 \mathrm{~min}$. All the swim sessions were videotaped and manually tracked using AnyMaze software keys. Only day 2 data are represented.

\section{Statistics}

The Gaussian distribution of the values was tested on Prism software using d'Agostino and Pearson or Shapiro-Wilk for $n<7$. If data followed a Gaussian distribution, two tailed $t$ test or one-way ANOVA test were performed; otherwise specific tests were applied as specified in the text. All results are expressed in mean \pm sem. $* p<0.05, * * p<0.01$ and $* * * p<0.005$.

\section{Results}

\section{Early-life stress (ELS) alters the expression of immediate early genes and genes involved in myelin formation in the developing medial prefrontal cortex (mPFC)}

As a preclinical model of ELS, we used the common model of chronic maternal separation, where pups are daily separated for $3 \mathrm{~h}$ from their dam starting at postnatal day 2 (P2) and until P14 (Fig. 1a) [11, 15]. This procedure has previously been shown to cause behavioural phenotypes in adults, such as increased depressive-like symptoms and cognitive deficits, suggestive of alterations of mPFC development. To identify early molecular candidates potentially involved in the permanent alterations induced by ELS, we focused on the chronic effects of maternal separation by analysing P15 brains, sampled $24 \mathrm{~h}$ after the last maternal separation (MS), compared with standard facility raised (SFR) pups. We performed an unbiased genetic screen using high-throughput RNA sequencing and minimised inter-individual variations and dissection biases by pooling $\mathrm{mPFC}$ of three males from different litters and testing four independent samples per condition. After normalisation, we found a total of 86 genes differentially expressed between MS and SFR animals (adjusted $p$-value $<0.05$ and change in transcript level $>30 \%$, Supplementary Table 1). Among the 56 upregulated genes, Gene Ontology (GO) analyses revealed a major enrichment in biological processes related to myelin ensheathment and oligodendrocyte differentiation (red dots in Fig. 1b and Supplementary Fig. 1a). Similar analyses of the 30 downregulated genes showed enrichment in cellular response to calcium (Supplementary Fig. 1b) likely due to a strong representation of several immediate early genes (IEG) (blue dots in Fig. 1b). To validate these changes, we performed quantitative PCRs on mPFC mRNAs obtained from a new cohort of MS and SFR animals analysed at P15. This second cohort confirmed the decrease in of several IEG transcripts (Fos, Fosb and Arc, Fig. 1c) and the increase in the myelin-related transcripts analysed (Mag, Mog and Plpl, Fig. 1d). We further examined whether increase in gene expression was reflected at protein levels. Western blots against PLP1 in the P15 mPFC showed an increase in PLP1 levels after MS $(0.45+/-0.07 ; n=5)$ compared to SFR $(0.27+/-0.06 ; n=5 ; p<0.01)$ (Supplementary Fig. 1c, d). Altogether, this unbiased approach identified early concomitant alterations of transcripts related to IEG and myelin ensheathment in the mPFC of postnatal animals exposed to maternal separation. 


\section{Premature OPC differentiation in ELS pups}

To determine possible causes of the increase in myelinrelated transcripts, we investigated oligodendrogenesis in the MS animals. The postnatal period corresponds to an intense proliferation of oligodendrocyte progenitor cells (OPCs) and P15 marks the initiation of differentiation into myelinating oligodendrocytes in the $\operatorname{mPFC}[20,29]$. We performed triple immunostaining of Olig2, PDGFR $\alpha$ and $\mathrm{CC} 1$ to distinguish $\mathrm{OPCs}\left(\mathrm{Olig} 2^{+} \mathrm{PDGFR}^{+}\right.$, Fig. 2a) from differentiated oligodendrocytes (OLs, Olig $2^{+} \mathrm{CC}^{+}$) [30]. At P15, the density of OPCs was unaltered in the mPFC of MS animals compared to SFR animals $(F(1,7)=0.032 p=$ 0.863 , Fig. $2 b$ ), but we detected a significant increase in the
Fig. 2 Maternal separation induces precocious

differentiation of

oligodendrocytes in the developing medial prefrontal cortex (mPFC). a The scheme illustrates the specificity of the oligodendrocyte lineage markers using triple immunostaining. Oligodendrocyte progenitor cells (OPCs) express Olig2 and high levels of PDGFR $\alpha$ while mature oligodendrocytes (OLs) express Olig2 and high levels of the $\mathrm{CC} 1$ antigen. Scale bar represents $20 \mu \mathrm{m}$. b-e Graphs show the density of OPCs $(\mathbf{b}, \mathbf{d})$ and OLs (c, e) in the P15 (b, c $n=5$ animals per conditions) and adult (d, $\mathbf{e} n=5$ animals per condition) mPFCs of SFR and MS animals. f Scheme shows the protocol of sequential labelling of OPCs using IdU injections at P10 and BrdU injections at P15 to differentiate the fraction of P10 OPCs that are still cycling at P15 (blue, Olig $2^{+} \mathrm{IdU}^{+} \mathrm{BrdU}{ }^{+}$) from those which have started differentiation by then (purple, Olig2 ${ }^{+} \mathrm{IdU}^{+} \mathrm{BrdU}^{-}$). g Graph shows a decresased fraction of cycling OPCs and an increased fraction of differentiated OPCs in P15 mPFC of MS animals as compared to SFR animals $(n=$ 11 SFR animals and $10 \mathrm{MS}$ animals). ${ }^{*} p<0.05,{ }^{* *} p<0.01$, $* * * p<0.005$ a

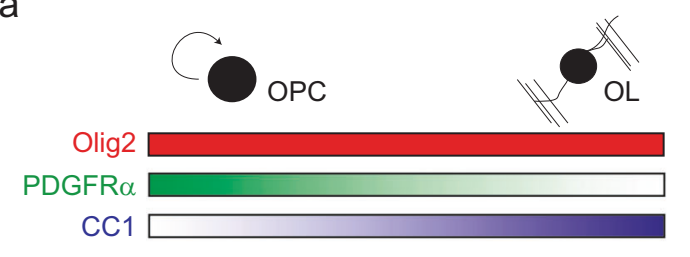

OPC

PDGFR $\alpha+$ Olig2+/mm2
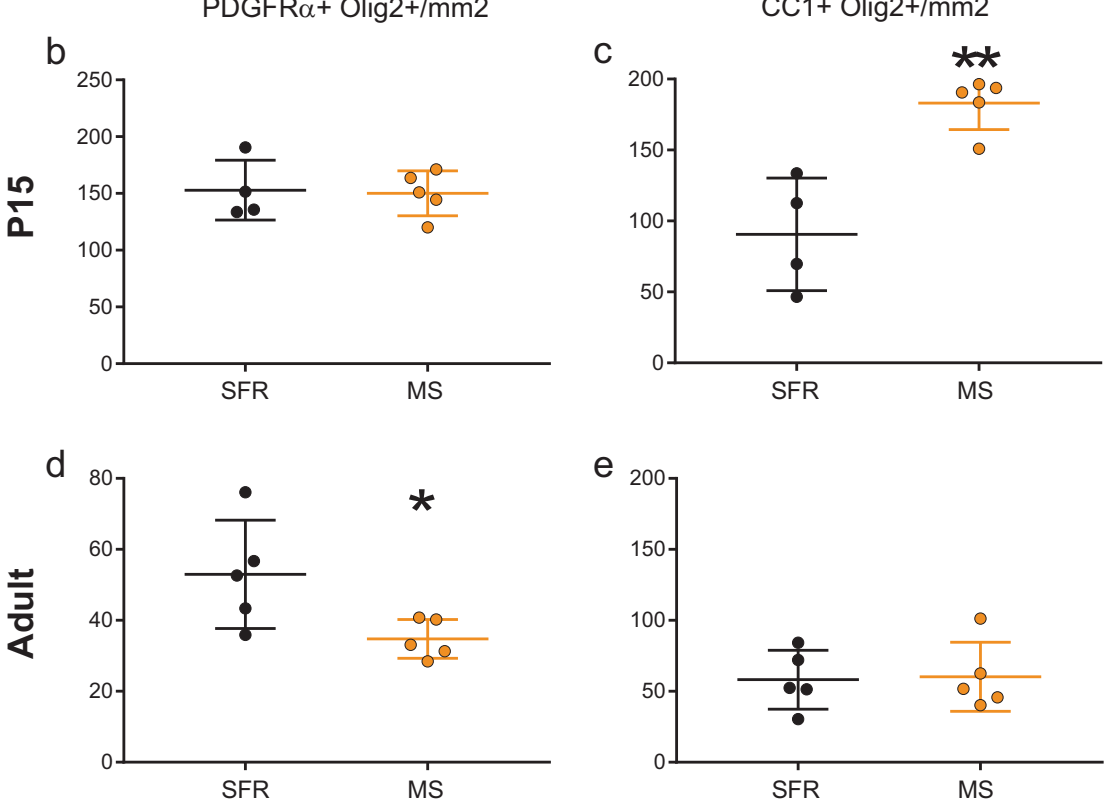

f

g
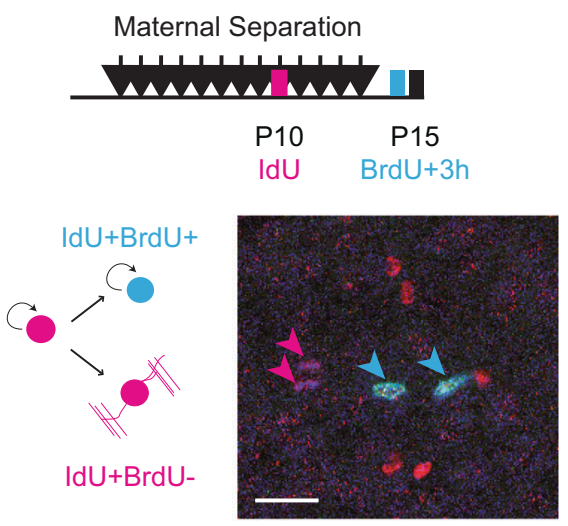

Olig2 PDGFR $\alpha$ CC1

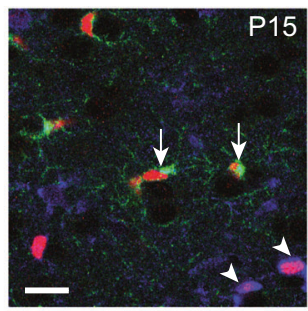

$\mathrm{OL}$

$\mathrm{CC} 1+$ Olig2+/mm2

P15 Olig2/ldU/BrdU per mm2

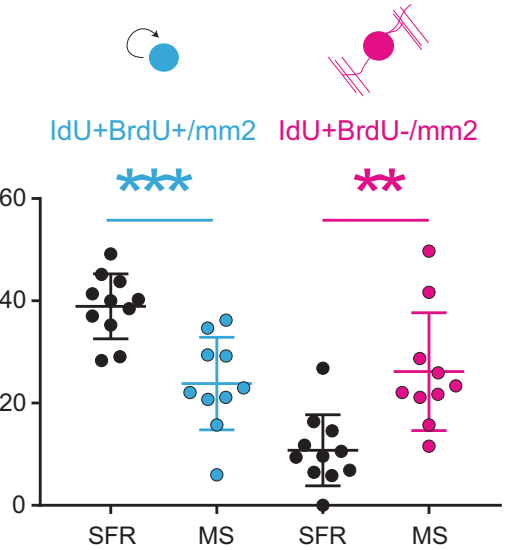


density of OLs $(F(1,7)=21.84 p=0.0023$, Fig. 2c). At adult stages, we observed a significant decrease in OPC density $(F(1,8)=6.28 p=0.036$, Fig. $2 d)$, while no difference in OL density or Plp1 levels was detected after MS $(F(1,8)=0.022 p=0.8865$, Fig. 2e and Supplementary Fig. 1d). To test whether early differentiation contributes to these observations, sequential staining of the dividing progenitors was performed using two thymidine analogues at P10 (iodo-deoxyuridine, IdU) and P15 (bromo-deoxyuridine, BrdU, Fig. 2f). This interval was chosen because postnatal OPCs differentiate into OLs in $\sim 5$ days [29]. In the mPFC of P15 MS animals, the fraction of OPCs that kept dividing between $\mathrm{P} 10$ and P15 (triple labelled with Olig $2^{+} \mathrm{IdU}^{+} \mathrm{BrdU}^{+}$, blue dots in Fig. $2 \mathrm{~g}, F(1,5)=12.62$ $p=0.0163$ ) was significantly reduced, while the differentiating fraction $\left(\mathrm{Olig} 2^{+} \mathrm{IdU}^{+} \mathrm{BrdU}^{-}\right.$, pink dots, $F(1,5)=$ $7.234 p=0.0433$ ) was increased. Thus, our results demonstrate that maternal separation induces a precocious differentiation of the OPC pool during development, as a potential cause for depletion of the OPC pool in adults. To assess how oligodendrogenesis defects could be related with the alterations in IEG we previously observed, we performed immunostainings on $\mathrm{MS}$ P9 $\mathrm{mPFC}$
(Supplementary Fig. 2). We observed that the decrease in cFos expression was already present at $\mathrm{P} 9$, while no change in $\mathrm{CC}^{+}{ }^{+} \mathrm{Olig} 2^{+}$or $\mathrm{PDGFR}^{+} \mathrm{Olig} 2^{+}$density could yet be detected. These results suggest that the chronic downregulation of neuronal activity in the MS pups preceded the defects in oligodendrogenesis. Considering the important role of neuronal activity in adult myelination [21], we further tested how manipulation of neuronal activity over the early postnatal period could modify oligodendrogenesis.

\section{Chemogenetic manipulation of mPFC postnatal activity bi-directionally controls developmental oligodendrogenesis}

We used viral delivery of AAVs expressing DREADDs to transiently and bi-directionally manipulate neuronal activity in the developing mPFC [22, 31]. AAV8-hSyn-hM4DimCherry or AAV8-hSyn-hM3Dq-mCherry viral constructs were bilaterally injected into the mPFC of all P1 pups (Fig. 3a). A time course analysis of DREADD expression in the $\mathrm{mPFC}$ showed mCherry expression visible as early as P2, with a progressive increase until P6 when expression reached a plateau (Fig. 3b). The characterisation of cell
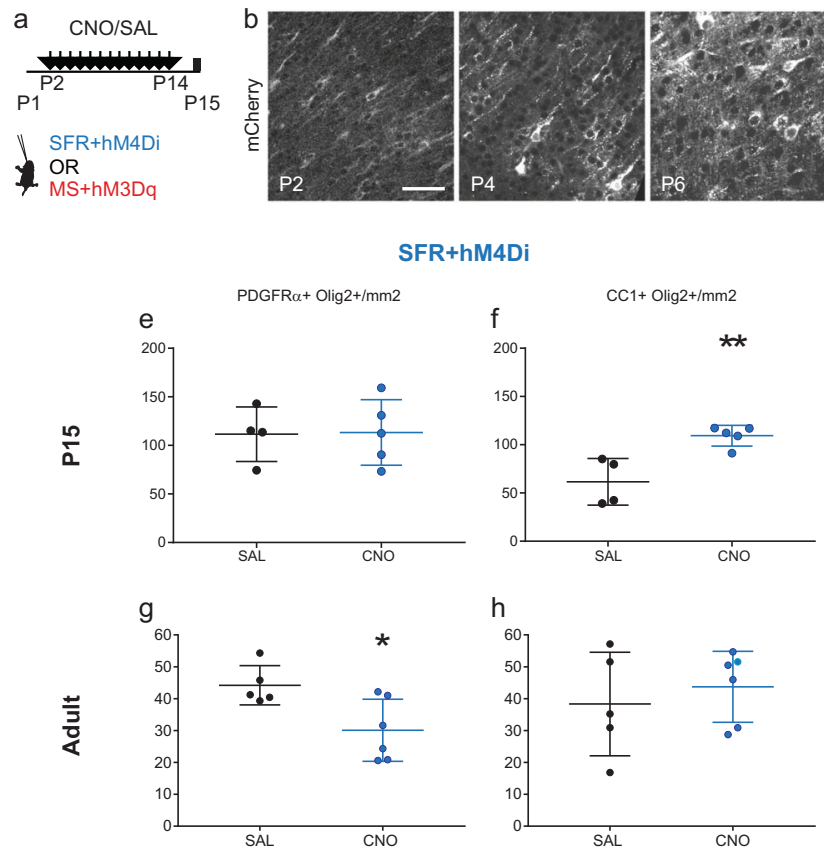

Fig. 3 Neuronal activity bi-directionally controls oligodendrocytes differentiation in the developing medial prefrontal cortex (mPFC). a Scheme illustrates the experimental paradigms of the chronic treatment with CNO or SAL solutions from P2 to P14 after local and bilateral injections at P1 of viral constructs expressing DREADDs and fused mCherry. All animals were either injected with the inhibitory hM4D (Gi) virus and raised under standard facility protocol (blue, SFR + $\mathrm{hM} 4 \mathrm{Di})$, or injected with excitatory $\mathrm{hM} 3 \mathrm{D}(\mathrm{Gq})$ virus and maternally separated (red, MS + hM3Dq). b Time course of DREADDs expression in the postnatal $\mathrm{mPFC}$ as observed by mCherry labelling. c, d

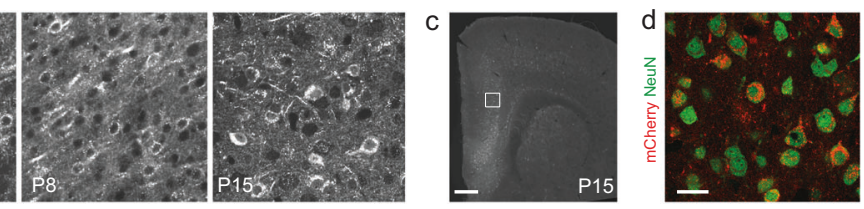

MS+hM3Dq
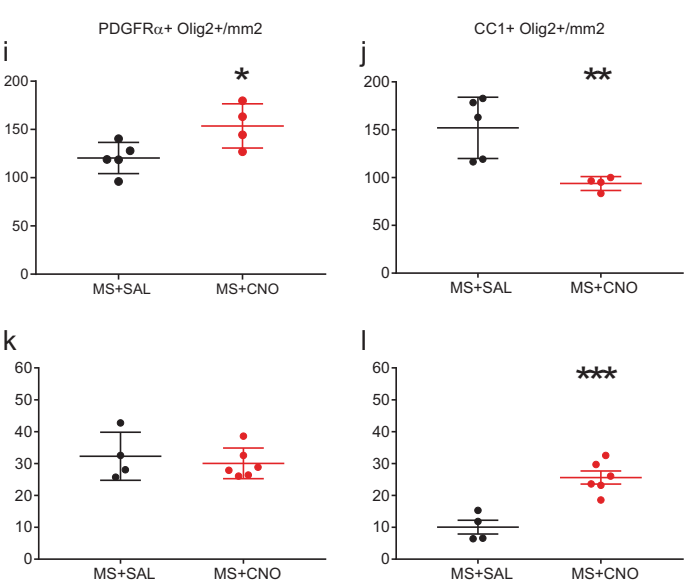

Micrographs show that transfection of the constructs was restricted to neuronal lineage as indicated $\mathrm{NeuN}$ and mCherry co-labelling (d) in P15 brain sections. $\mathbf{e}-\mathbf{h}$ Graphs show the density of OPCs $(\mathbf{e}, \mathbf{g})$ and OLs (f, h) at P15 (e, f, $n=4 \mathrm{SAL} / 5 \mathrm{CNO})$ and in adults (g, h, $n=$ 5SAL/6CNO) mPFCs of SFR + hM4Di. i-l Graphs show the density of OPCs (i, k) and OLs (j, l) at P15 (i, j $n=5 \mathrm{SAL} / 4 \mathrm{CNO})$ and in adults $(\mathbf{k}, \mathbf{l} n=4 \mathrm{SAL} / 6 \mathrm{CNO}) \mathrm{mPFCs}$ of MS $+\mathrm{hM} 3 \mathrm{Dq}$ animals. Scale bars represent $50 \mu \mathrm{m}(\mathbf{b}), 500 \mu \mathrm{m}(\mathbf{c})$ and $20 \mu \mathrm{m}(\mathbf{d}) .{ }^{*} p<0.05$, $* * p<$ $0.01, * * * p<0.005$ 
types transfected was done at P15: $38 \% \pm 5.2$ of the mPFC $\mathrm{NeuN}^{+}$cells were mCherry ${ }^{+}$upon hM4Di injections and $51.8 \% \pm 4.4$ upon $\mathrm{hM} 3 \mathrm{Dq}$ injections. In addition, $95.98 \% \pm 0.06$ of the mCherry ${ }^{+}$cells were $\mathrm{NeuN}^{+}$, and no co-expression of mCherry with Olig2 or PDGFR $\alpha$ or the oligodendrocyte-specific antibody $\mathrm{CC} 1$ could be detected (data not shown) supporting that our viral transfection experiments essentially targeted neurons. A bias for deeplayer glutamatergic neurons was observed using Tbr1 and $\mathrm{Ctip}^{+}$as markers of these populations: $72.59 \% \pm 3.75$ of the mCherry $^{+}$cells were $\mathrm{Tbr} 1^{+}$or $\mathrm{Ctip}^{+}$and only $6.17 \% \pm 1.24$ were $\mathrm{GABA}^{+}$(Fig. 3b, c).

All mice received viral injections in the PFC at P1, and further received daily injections of saline (SAL) or the inert ligand Clozapine-N-Oxyde (CNO) from P2 to P14. Pups injected with the inhibitory construct were raised in the SFR condition (referred to as SFR + hM4Di, Fig. 3a). Conversely, pups injected with the activating construct were submitted to the maternal separation protocol (referred to as MS + hM3Dq).

To evaluate how these experimental conditions modified PFC activity, we used ex vivo patch-clamp electrophysiological recordings to measure neuronal excitability and immunolabeling of immediate early genes (cFos/ Egr1) to evaluate the in vivo consequences on mPFC neural activity. We first tested the effect of DREADD activation on intrinsic excitability, i.e., the propensity of a neuron to fire action potentials when subjected to an input current. In slices of animals receiving mPFC hM3Dq injections, there was a robust increase in neuronal intrinsic excitability in the $\mathrm{hM} 3 \mathrm{Dq}-\mathrm{mCherry}{ }^{+}$layer five pyramidal neurons upon exposure to $\mathrm{CNO}(30 \mu \mathrm{M})$ or to its potential metabolite clozapine $(1 \mu \mathrm{M})$ (Supplementary Fig. 3a, b). In parallel, a significant increase in cFos labelling in the mPFC was observed (Supplementary Fig. 4c). In slices prepared from pups with the SFR + hM4Di protocol, exposure to CNO or clozapine did not modify intrinsic excitability significantly (Supplementary Fig. $3 \mathrm{c}, \mathrm{d}$ ), although the low firing frequency and the resulting high trial-to-trial variability in our experimental conditions may have made small effects difficult to detect. Since hM4Di activates $\mathrm{G}$ protein-coupled inwardly rectifying potassium (GIRK) channels, we tested whether CNO elicits GIRK-mediated currents in voltage-clamped hM4Di-mCherry ${ }^{+}$pyramidal neurons. Indeed, CNO application $(30 \mu \mathrm{M})$ elicited a small but significant outward current $(16.3 \pm 5.8 \mathrm{pA}, \quad n=11, \quad p=0.002$, Mann-Whitney test) that was inhibited by barium $(100 \mu \mathrm{M})$, a blocker of GIRK channels (Supplementary Fig. 3e, f). As recent results suggested that hM4Di was pre-synaptically targeted in cortical neurons [32], we examined whether hM4Di also altered the probability of glutamate release from pyramidal neurons. We expressed both Channelrhodopsin (ChR2-YFP) and hM4Di-mCherry in mPFC under the control of the synapsin promoter. In ChR2-YFP- hM4Di-mCherry ${ }^{-}$ layer five thick-tufted neurons located in the vicinity of $\mathrm{ChR}$ - $\mathrm{YFP}^{+} \mathrm{hM}^{+} \mathrm{Di}-\mathrm{mCherry}{ }^{+}$neurons, short-light pulses elicited excitatory postsynaptic currents (EPSCs, Supplementary Fig. 3i). The paired-pulse ratio, a measure of release probability, was unchanged in $\mathrm{CNO}$-exposed neurons versus controls (respectively, $0.982 \pm 0.009$ and $0.985 \pm 0.006, p=0.81$, two-tailed $t$-test, (Supplementary Fig. 3j). Thus, hM4Di activation inhibited the somatodendritic compartment of pyramidal cells through GIRK channel activation but did not modify presynaptic release. The inhibitory action of hM4Di was also confirmed by a significant decrease in mPFC Egr1 expression among hM4Di-mCherry ${ }^{+}$neurons in P8 and P15 animals sacrificed $2 \mathrm{~h}$ after CNO injection (Supplementary Fig. 4a). Additionally, a sustained decrease in cFos labelling was observed in P15 mPFC of SFR + hM4Di mice sacrificed $24 \mathrm{~h}$ after chronic $\mathrm{CNO}$ exposure (P2-P14) compared to the saline treated group (Supplementary Fig. 4b). Altogether, these results support the efficacy of our pharmacogenetic approach for either mimicking or counter-acting the chronic reduction in IEG expression in the MPFC induced by MS during the postnatal period.

To test the role of changes in early neuronal activity in the oligodendrogenesis defects induced by maternal separation, P15 and adults brains were analysed after SFR + hM4Di or MS + hM3Dq treatments, as described above. In P15 SFR + hM4Di animals exposed to CNO, we found no difference in OPC density but an increased density of OLs compared to saline exposed animals $(F(1,7)=15.991 p=0.005$, Fig. 3f $)$; while at adult stages, we observed a decrease in OPC density $(F(1,9)=7.809$ $p=0.0209$, Fig. $3 g$ ) with no changes in OL density in $\mathrm{CNO}$ versus SAL groups. Conversely, in the P15 MS + $\mathrm{hM} 3 \mathrm{Dq} \mathrm{mPFC}$, we found an increase in OPC density $(F(1,7)=6.52 p=0.0379$, Fig. $3 i)$, with no changes in OL density in CNO versus SAL group comparisons (Fig. 3j). In adults that had been developmentally exposed to $\mathrm{MS}+\mathrm{hM} 3 \mathrm{Dq}$, there was an increase in OL density $(F(1,8)=25.795 p=0.001$, Fig. 31) with no alterations in OPC density when comparing the CNO versus SAL groups (Fig. 3k). Overall, this indicated that transient reduction of $\mathrm{mPFC}$ neuron excitability reproduced the oligodendrogenesis defects of MS mice, suggesting a similar premature exit of the cell cycle. Moreover, increasing neuronal excitability of $\mathrm{mPFC}$ neurons was sufficient to prevent the oligodendrogenesis defects induced by MS. These results indicate a causal role of altered $\mathrm{mPFC}$ neuronal activity on the precocious oligodendrogenesis observed in MS animals. 


\section{Postnatal local decrease in mPFC activity recapitulates $M S$ behavioural phenotype in adults}

We then tested whether chemogenetic manipulations in the developing PFC could affect the emotional and cognitive symptoms induced by MS. We began with a characterisation of the behavioural phenotype of adult MS BALBcJ mice (Fig. 4a), since some variability of long-term behavioural effects of MS have been reported between different mouse strains [11]. In our protocol, adult MS males showed decreased exploration (Mann-Whitney $p=0.0002$ ) and time spent in the centre in the open field $(\mathrm{OF}, F(1,25)=4.317$ $p=0.0482$, Fig. $4 \mathrm{~b}, \mathrm{c})$ as well as decreased time spent in the open arm of the elevated plus maze (EPM, $F(1,22)=5.438$ $p=0.0293$, Fig. 4d). They further showed an increased burying behaviour in the marble test $(F(1,32)=5.402 p=$ 0.0266, Fig. 4e), when compared with SFR animals. In addition, MS males displayed an increased time floating in
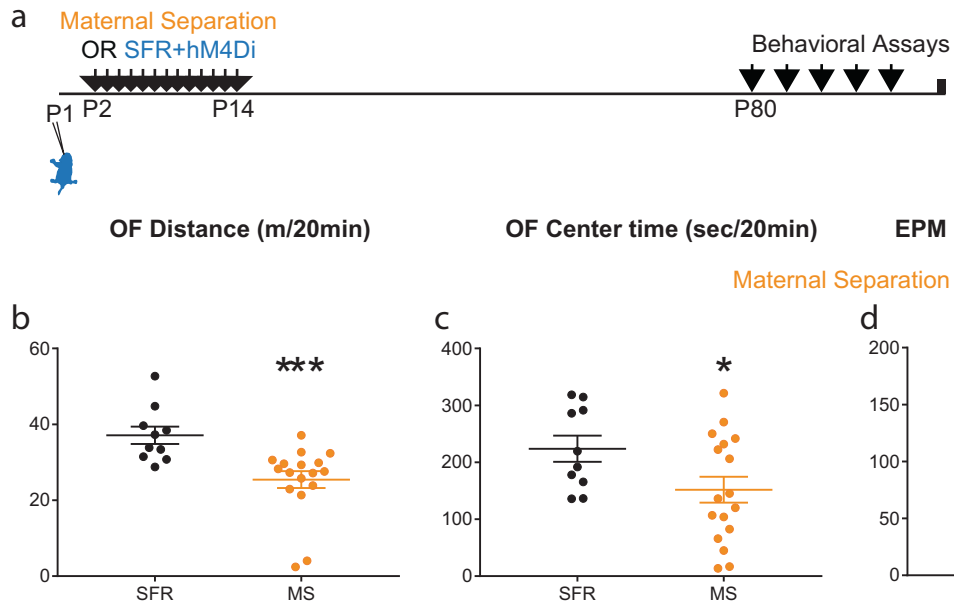

EPM Open arm time (sec/10min)

\# Marbles burried (per min)
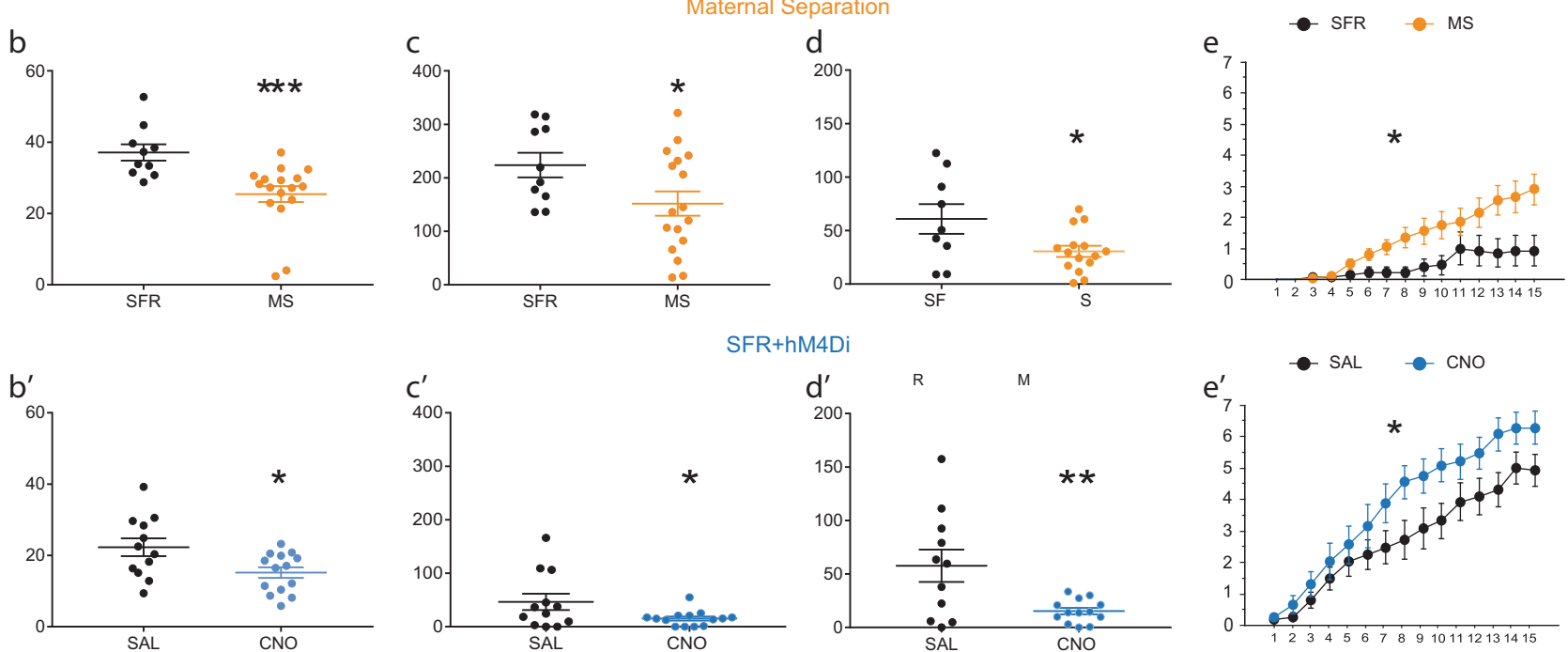

FST Floating time (sec/min)
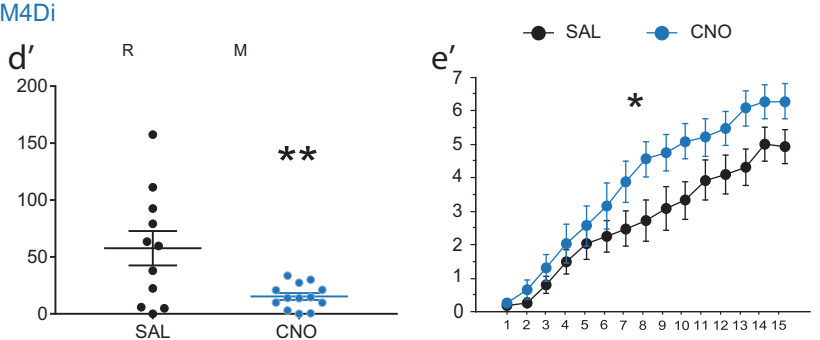
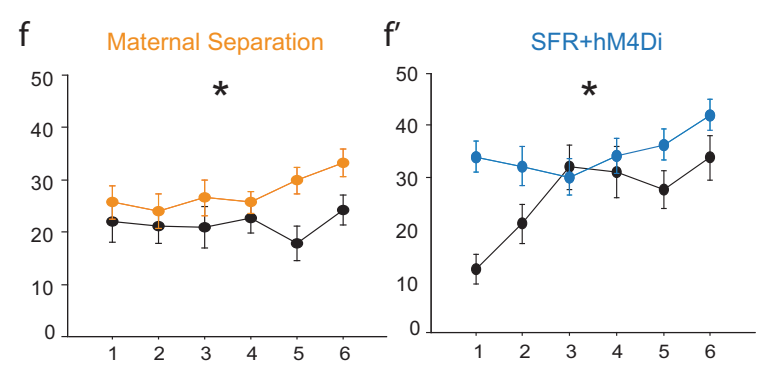

9

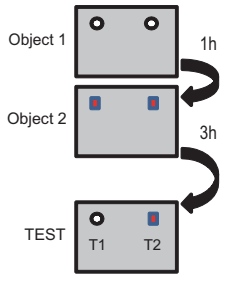

Sequential object preference

$(\mathrm{T} 1-\mathrm{T} 2) * 100 /(\mathrm{T} 1+\mathrm{T} 2)$

$\mathrm{g}^{\prime}$

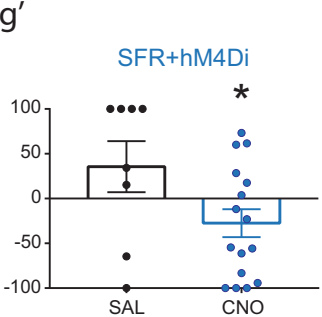

Fig. 4 Early inhibition of medial prefrontal cortex (mPFC) neurons recapitulates the emotional disorders induced by early-life stress. a Scheme illustrates the experimental paradigms of maternal separation (orange) or SFR + hM4Di (blue) treatments followed by behavioural testing in adulthood. b, b' Graphs show the distance travelled in an open field (OF) by SFR or MS animals (b, $n=$ 10 SFR/17MS) and by SFR + hM4Di animals exposed to chronic SAL or CNO (b', $n=12 \mathrm{SAL} / 14 \mathrm{CNO})$. c, $\mathbf{c}^{\prime}$ Graphs show the time spent in the centre of the OF during the same 20 min test. d, d' Graphs show the time spent in the open arm of an elevated plus maze (EPM) during a $10 \mathrm{~min}$ test $(n=9 \mathrm{SFR} / 15 \mathrm{MS}$ animals and $n=11 \mathrm{SAL} / 13 \mathrm{CNO})$. e, $\mathbf{e}^{\prime}$ Graphs show the number of buried marbles at each minutes of a $15 \mathrm{~min}$ test $(n=14 \mathrm{SFR} / 20 \mathrm{MS}$ and $n=13 \mathrm{SAL} /$ $15 \mathrm{CNO}$ animals). f, $\mathbf{f}^{\prime}$ Graphs show the time floating per min of a $6 \mathrm{~min}$ forced swim test (FST, $n=10 \mathrm{SFR} / 18 \mathrm{MS}$ and $n=12 \mathrm{SAL} /$ $16 \mathrm{CNO})$. $\mathbf{g}$, g' Scheme represents the experimental paradigm of the sequential object recognition test with $3 \mathrm{~min}$ exposure to two identical object 1 , followed $1 \mathrm{~h}$ later by $3 \mathrm{~min}$ exposure to two identical object 2 , and $3 \mathrm{~h}$ later by $4 \mathrm{~min}$ exposure to object 1 and object 2. Graphs indicate the preference ratio for object $1(n=14 \mathrm{SFR}$ $/ 10 \mathrm{MS}$ animals and $n=8 \mathrm{SAL} / 15 \mathrm{CNO}$ animals). $* p<0.05$, ${ }^{* *} p<$ $0.01, * * * p<0.005$ 
the forced swim test (FST, $F(1,26)=6.048 p=0.0209$, Fig. 4f) and an impaired short-term memory in the sequential novel object recognition test $(\mathrm{SNOR}, F(1,23)=4.75 p=$ 0.0396 ,) which measures temporal order memory (as the preference in exploring an object seen less recently than another) $[18,28]$. These results confirmed an increase in anxiety and depression-related behaviours in MS animals and an impairment in a test of recognition memory, which has been linked to mPFC function [28].

In adults SFR + hM4Di animals, comparison of mice exposed to CNO or SAL from P2 to P14 revealed behavioural alterations very similar to those of MS animals. These included reduced exploration in the OF (distance $F(1,24)=6.433 p=0.0181$, Fig. $4 b^{\prime}$ and centre time $F(1,24)=4.46 p=0.0452$, Fig. $\left.4 \mathrm{c}^{\prime}\right)$, reduced exploration in the EPM $(F(1,22)=9.94 p=0.0067$, Fig. 4d'), increased marble burying $(F(1,26)=5.233 p=0.305$, Fig. 4e'), increased immobility in the FST $(F(1,26)=5.675 p=$ 0.0248 , Fig. $\left.4 \mathrm{f}^{\prime}\right)$ and decreased preference in the SNOR test $\left(F(1,19)=4.443 p=0.0485\right.$, Fig. $\left.4 \mathrm{~g}^{\prime}\right)$. They also show delayed postnatal growth with a sustained decrease in adult weight, as MS animals (Supplementary Fig. 5). To exclude any unwarranted effects of the effects of chronic exposure to CNO, we injected a new cohort of animals with AAV8hSyn-GFP at P1, administered CNO or SAL daily from P2 to P14 and tested their adult behaviour. In this cohort, no change in any of the behavioural tests was found between the CNO and SAL groups (Supplementary Fig. 6). Altogether, our data show that decreasing mPFC neuronal activity during development is sufficient to recapitulate salient features of the MS's adult phenotype.

\section{Postnatal local increase in mPFC activity prevents the emergence of depression-related phenotype induced by maternal separation}

To test the role of reduced mPFC activity in the behavioural phenotype of MS animals, we assessed the behaviour of animals exposed to MS with or without chemogenetic activation (hM3Dq) of mPFC neurons (Fig. 5a). CNO- and

a

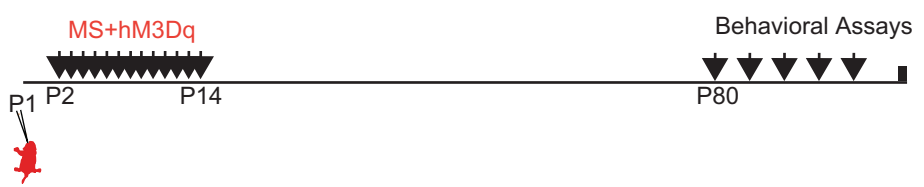

b

OF Distance (m/20min)

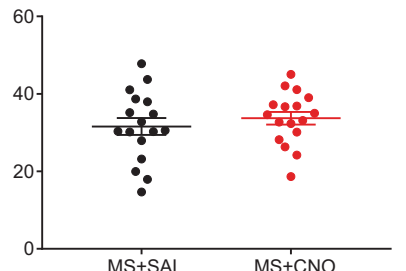

f FST Floating time (sec/min)

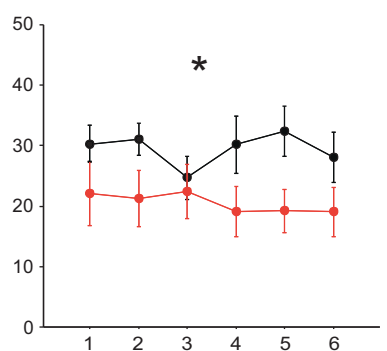

C OF Center time (sec/20min)

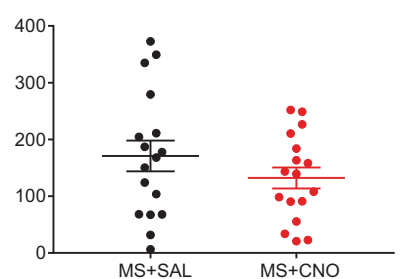

g

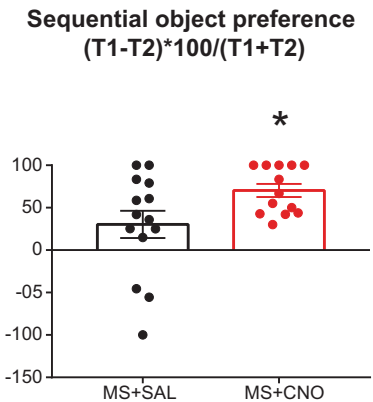

d EPM Open arm time (sec/10min)

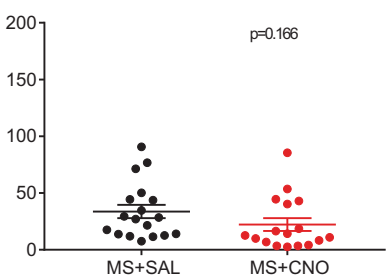

h

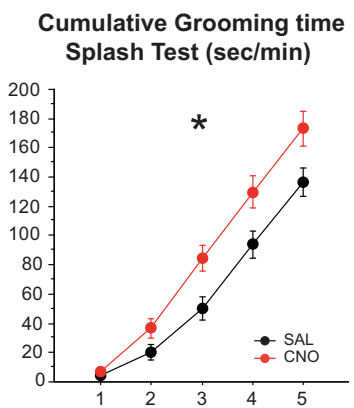

e \# Marbles burried (per min)

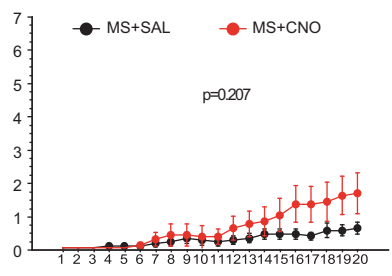

i Cumulative Grooming time Splash Test (sec/min)

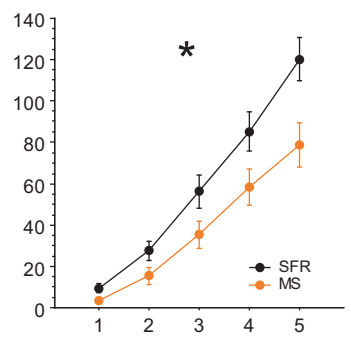

Fig. 5 Early activation of medial prefrontal cortex (mPFC) neurons prevents the emergence of the depression-like phenotype induced by early-life stress. a Scheme illustrates the experimental paradigms of MS + hM3Dq animals, analysed for behaviour as adults. b Graph shows the distance travelled in an open field (OF) by MS + hM3Dq animals exposed to SAL (black, $n=17$ ) or CNO (red, $n=17$ ). c Graph shows the time spent in the centre of the OF during the same 20 min test ( $n=17 \mathrm{SAL} / 17 \mathrm{CNO})$. d Graph shows the time spent in the open arm of an EPM during a 10 min test ( $n=18$ SAL/17CNO).

e Graph shows the number of buried marbles at each minutes of a 20 min test ( $n=18 \mathrm{SAL} / 16 \mathrm{CNO}$ ). f Graph shows the time floating per min during a $6 \mathrm{~min}$ FST. $g$ Graph shows the preference ratio for Object 1 ( $n=14 \mathrm{SAL} / 13 \mathrm{CNO})$. h, i Graphs show the cumulative time grooming after a splash with $20 \%$ sucrose solution. Comparison was made between SFR $+\mathrm{hM} 3 \mathrm{Dq}$ animals exposed to chronic SAL or $\mathrm{CNO}(\mathrm{H}, n=14 \mathrm{SAL} / 13 \mathrm{CNO})$ and between SFR or MS animals (I, $n=15 \mathrm{SAL} / 12 \mathrm{CNO}) . * p<0.05$ 
SAL-treated MS mice showed no difference in the behavioural parameters related to anxiety including: the distance or the time in the centre of the OF (Fig. 5b, $F(1,32)=0.604$ $p=0.4427$ and $C, F(1,32)=1.397 p=0.246)$, the time in the open arm of the EPM (Fig. 5d, $F(1,33)=2.002 p=$ 0.1664 ) or the number of burying in the marble test (Fig. 5e, $F(1,32)=1.656 \quad p=0.2074)$. However, MS + hM3Dq animals exposed to CNO floated less in the FST (Fig. 5f, $F(1,29)=6.508 p=0.0163)$ and had improved performance in the temporal recognition memory-test (Fig. 5g, Mann-Whitney $p=0.0463$ ). To strengthen our analysis of the depression-related phenotype we used the sucrose splash test, which measures self-care and hedonic behaviours [33]. Animals solely exposed to MS display a decrease in grooming when compared with SFR animals (Fig. 5I, $F(1,25)=5.618 p=0.0258)$. We observed an increased grooming behaviour in CNO-exposed $\mathrm{MS}+$ hM3Dq animals as compared to SAL (Fig. 5h, $F(1,25)=$ $6.532 p=0.171)$. Hence, transient increase in $\mathrm{mPFC}$ neuronal excitability prevented the emergence of the depression and recognition memory defects that characterise MS exposed mice, but had no effect on the anxiety-related phenotypes.

\section{Discussion}

The present study identified alterations in the expression of genes related to neuronal activity and to myelination in the developing mPFC of mice exposed to early-life stress. We found that chronic maternal separation induced a precocious differentiation of the oligodendrocyte lineage in pups, together with a subsequent depletion of the OPC pool in adults. Chemogenetic tools demonstrated that these alterations are controlled by local changes in mPFC neuronal activity during early postnatal development. Furthermore, we established that bidirectional changes of neuronal activity in the developing $\mathrm{mPFC}$ are sufficient to modulate adult behaviours, and in addition, to mimic or rescue the long-term effects of early-life stress.

\section{The pre-weaning period is critical for $\mathrm{mPFC}$ myelination}

Early-life stress in humans has been linked with hypomyelination in the adult prefrontal cortex [5-8], a phenotype also reported in rodent models of ELS [9-12, 20]. Present findings provide a possible developmental mechanism for this, by showing that a precocious differentiation of the oligodendrocyte lineage during development could be at the origin of the permanent reduction in the adult OPC pool. Thus, the first 2 postnatal weeks appear to represent a critical period for oligodendrogenesis, with an activity-dependent regulation of maturation and cell cycle exit that has long-term effects on the adult progenitor pool. This could add up to later critical periods for oligodendrogenesis identified in juveniles, where social isolation (P21-P35) also induced a sustained decrease in myelin content and immediate early gene's expression, which correlate with a permanent impairment in social behaviour at adult stages, although the developmental mechanisms were unclear [34]. Importantly, developmental exposure to stress contrasts with the effects of adult exposure to chronic stress, which has only transient consequences on mPFC myelination and behaviour [34-36]. Therefore, our results, by showing the importance of the P2-P14 period, suggest that successive and/or expanded critical period(s) occur in the mPFC to establish mPFC myelination, and correlate with alterations in the functions of anxiety, mood and social circuits.

The exact developmental mechanisms involved in adult hypomyelination after ELS are still disputed [7, 12]. Our data favour a precocious differentiation of the oligodendrocyte lineage resulting in a depletion of the OPC pool in adults. In another study, Yang et al. [20] reported a deficit in OPC differentiation in the MPFC of MS Sprague Dawley rats. The discrepancy with the present results is unclear and could be due to a number of technical differences between the studies such as delayed timing of analysis (P15 in mice vs. P21 in rats), and the differences in markers of proliferation that were used. Importantly, our findings are supported by a transient increase in PLP1 levels as well as by several previous observations in other experimental models of sensory deprivation or ELS, showing transient and immediate increase in myelin content, followed by a permanent decrease in adulthood in sensory cortices or cerebellum [37-41]. Moreover, a recent clinical paper indicated that childhood adversity in human biases oligodendrocyte lineage toward a more differentiated phenotype in the ventral $\mathrm{mPFC}$, with more pronounced effects in samples from young individuals that died closer to ELS experience [7]. Altogether, these results suggest that early stressors induce a pathological precocious differentiation of OLs, which thereafter alters myelin content, or the capacity of myelin plasticity in adult life.

\section{Neuronal activity controls early stages of oligodendrogenesis}

Our unbiased transcriptomic approach identified an important decrease of several immediate early genes in the P15 $\mathrm{mPFC}$ after maternal separation, as a proxy for a deficit in neuronal plasticity [42]. The converging reduction of Fos and $F o s B$ further suggest that there is a chronic dampening of neural network activity in the mPFC of pups undergoing chronic maternal separation experience. These results are in 
agreement with previous reports in the MS rat model at P21, showing a local decrease in specific immediate early gene expressions correlating with a reduced neuronal synchrony in the $\mathrm{mPFC}$, as revealed by in vivo recordings [14, 18]. Converging experimental evidence, provided in the present study, support a causal link between activity changes and the precocious differentiation of oligodendrocytes. On one hand, reduced cFos expression in the mPFC preceded the changes in OPCs differentiation (Supplementary Fig. 2), on the other hand, chemogenetic inhibition of the mPFC in the early postnatal period induced a bias toward OPC differentiation, thus mimicking the MS phenotype. Finally, increasing neuronal excitability prevented the bias toward OPC differentiation, induced by MS. To the best of our knowledge, this is the first demonstration of a causal role of neuronal activity on $\mathrm{mPFC}$ oligodendrogenesis during early postnatal life.

In adults, increased neuronal activity stimulates proliferation and differentiation of OPCs and increases myelination in motor cortices [21, 43, 44], while chronic stress decreases IEG expression and impairs OPC proliferation and myelination in the $\operatorname{mPFC}[34,45]$. This is opposite to the effect that we observe during early postnatal life, since we show that increased neuronal activity stimulates proliferation at the expense of differentiation. Therefore, it is likely that different activity-dependent regulations of oligodendrogenesis are at play during development. This hypothesis is also supported by changes in the molecular properties of OPCs during this postnatal period [46, 47], including the establishment of transient synaptic connectivity between OPCs and interneurons [21, 48]. It will be important in the future to determine how activity-dependent signalling differs during early postnatal life and adulthood to control OPC maturation and which are the cellular substrates involved.

\section{Early mPFC physiology and psychiatric disorders}

Another important finding of the present study is the direct role of postnatal mPFC activity in the aetiology of emotional and memory phenotypes induced by ELS. The mPFC neuronal network is still being assembled during the postnatal period, and previous studies have identified P2-P14 as a critical period for emotional and cognitive functions [49]. A critical role of neuronal activity in cortical maturation is well established in the primary sensory cortical maps (visual, somato-sensory, auditory) [50, 51]. Activity thus appears as the mechanistic link between changes in the environment and alterations in the brain's developmental trajectory. Our study provides the first evidence of an activity-dependent maturation of mPFC emotional circuits. An important question remains however open: determining the effects of ELS on neural circuit activity, which is presently unclear. Some recent investigations have started addressing this question with in vivo physiological recordings. Using this technique in P12-P17 rats, these studies showed that ELS decreased the power of low-frequencies oscillations in the mPFC and altered cortical synchrony $[18,52]$. These effects could be driven by changes in neuronal activity in different mPFC circuits, as suggested by another recent study showing that optogenetic activation of deep layers generates a global increase in mPFC oscillatory power in the neonatal mouse cortex (P7), while activation of the upper layers mainly drives beta oscillations [53]. These recent technical advances raise hope for future dissection of ELS pathophysiology and could help resolving whether altered maturation of specific mPFC circuits could underlie vulnerability to depression and anxiety disorders.

Our results also suggest that developmental mechanisms underlying anxiety- and depression-related behaviours differ, as mPFC activation in MS pups only restored the depression-related phenotype. The reason for this is unclear. Generally one may argue that restoring a normal phenotype requires more precision in the activation of spatial and temporal activity patterns, whereas inhibition is more crude. In addition, our viral infection showed a bias toward deep layers containing PFC-raphe connections important in depression-related phenotypes [54], whereas the PFCamygdala connectivity sustaining anxiety originate from both superficial and deep mPFC layers [55].

On a translational perspective, our results demonstrate that early mPFC activity tightly controls two major endophenotypes related to ELS, namely oligodendrogenesis and depression-related behaviour, thus supporting a common origin for these typical traits of emotional disorders [56-58]. Strikingly, most mental disorders, including emotional disorders and schizophrenia, involve alterations of mPFC myelination and the susceptibility to develop these disorders is increased by ELS $[56,59,60]$. The possible causal role of oligodendrogenesis in the aetiology of emotional disorders has been suggested by several recent studies. For instance, genetic alterations of oligodendrogenesis were correlated with emotional disorders in adult rodents [34, 61-65] and restoring myelination was sufficient to rescue the social avoidance induced by chronic stress [36]. Present results further support this notion, by showing that ELS alters mPFC oligodendrogenesis and emotional behaviours via shared activity-dependent mechanisms.

\section{Conclusion}

This study demonstrates that ELS leads to a dampening of immediate early gene expression in the developing $\mathrm{mPFC}$, resulting in a precocious maturation of the oligodendrocyte lineage and a subsequent reduction of adult myelin 
progenitors in adult life. Moreover, our results show that early postnatal changes in $\mathrm{mPFC}$ neuronal activity in mouse pups play a crucial role in determining the emotional and temporal memory alterations that emerge in adulthood after early-life stress events.

Acknowledgements We thank Dr. Macklin, Dr. Soiza-Reilly, Dr. Parras and Dr. Angulo for their scientific and technical advices and comments on the manuscript. We are also thankful to the members of the CEF UMS28 facility as well as the U839 and U894 imaging platforms for their support.

Funding The work was funded by the Labex BioPsy, the French Agence Nationale pour la Recherche (ANR-11-0004-02; ANR-160162), the DIM Région Ile de France, and the Behaviour and Brain Foundation Young Investigator-NARSAD Grant. BAdC received a scholarship from Coordination for the Improvement of Higher Education Personnel (CAPES), Brazil. JO is the recipient of the Ecole des Neurosciences de Paris (ENP) scholarship. AT is a CNRS (Centre National de la Recherche Scientifique) investigator.

Author contributions AT generated the cohorts, performed the histological and behavioural experiments and analysed all the data. JO and BLSAdC provided help for the preparation of animal cohorts and histological analysis. CL-M and AMDS performed the electrophysiological recordings and analysed the data. YL performed and analysed the transcriptome profiling experiments. AB and VAV helped to design the study. PG and AT designed the study and wrote the manuscript with the help of all authors.

\section{Compliance with ethical standards}

Conflict of interest The authors declare that they have no conflict of interest.

Publisher's note: Springer Nature remains neutral with regard to jurisdictional claims in published maps and institutional affiliations.

Open Access This article is licensed under a Creative Commons Attribution 4.0 International License, which permits use, sharing, adaptation, distribution and reproduction in any medium or format, as long as you give appropriate credit to the original author(s) and the source, provide a link to the Creative Commons license, and indicate if changes were made. The images or other third party material in this article are included in the article's Creative Commons license, unless indicated otherwise in a credit line to the material. If material is not included in the article's Creative Commons license and your intended use is not permitted by statutory regulation or exceeds the permitted use, you will need to obtain permission directly from the copyright holder. To view a copy of this license, visit http://creativecommons. org/licenses/by/4.0/.

\section{References}

1. Norman RE, Byambaa M, De R, Butchart A, Scott J, Vos T. The long-term health consequences of child physical abuse, emotional abuse, and neglect: a systematic review and meta-analysis. PLoS Med. 2012;9:e1001349.

2. Pechtel P, Pizzagalli DA. Effects of early life stress on cognitive and affective function: an integrated review of human literature. Psychopharmacol (Berl). 2011;214:55-70.
3. Kessler RC, McLaughlin KA, Green JG, Gruber MJ, Sampson NA, Zaslavsky AM, et al. Childhood adversities and adult psychopathology in the WHO World Mental Health Surveys. Br J Psychiatry. 2010;197:378-85.

4. Heim C, Shugart M, Craighead WE, Nemeroff CB. Neurobiological and psychiatric consequences of child abuse and neglect. Dev Psychobiol. 2010;52:671-90.

5. Insana SP, Banihashemi L, Herringa RJ, Kolko DJ, Germain A. Childhood maltreatment is associated with altered frontolimbic neurobiological activity during wakefulness in adulthood. Dev Psychopathol. 2016;28:551-64.

6. Nelson CA. Hazards to early development: the biological embedding of early life adversity. Neuron. 2017;96:262-6.

7. Tanti A, Kim JJ, Wakid M, Davoli M-A, Turecki G, Mechawar N. Child abuse associates with an imbalance of oligodendrocyte-lineage cells in ventromedial prefrontal white matter. Mol Psychiatry. 2018;23:2018-28.

8. Syed SA, Nemeroff CB. Early life stress, mood, and anxiety disorders. chronic. Stress. 2017;1:247054701769446.

9. Gutman DA, Nemeroff CB. Neurobiology of early life stress: rodent studies. Semin Clin Neuropsychiatry. 2002;7:89-95.

10. Callaghan BL, Sullivan RM, Howell B, Tottenham N. The international society for developmental psychobiology Sackler symposium: early adversity and the maturation of emotion circuits-a crossspecies analysis. Dev Psychobiol. 2014;56:1635-50.

11. Tractenberg SG, Levandowski ML, de Azeredo LA, Orso R, Roithmann LG, Hoffmann ES, et al. An overview of maternal separation effects on behavioural outcomes in mice: evidence from a four-stage methodological systematic review. Neurosci Biobehav Rev. 2016;68:489-503.

12. Bordner Ka, George ED, Carlyle BC, Duque A, Kitchen RR, Lam TT, et al. Functional genomic and proteomic analysis reveals disruption of myelin-related genes and translation in a mouse model of early life neglect. Front Psychiatry. 2011;2:1-18.

13. Braun K, Bock J. The experience-dependent maturation of prefronto-limbic circuits and the origin of developmental psychopathology: implications for the pathogenesis and therapy of behavioural disorders. Dev Med Child Neurol. 2011;53:14-8.

14. Sood A, Pati S, Bhattacharya A, Chaudhari K, Vaidya VA. Early emergence of altered 5-HT2A receptor-evoked behavior, neural activation and gene expression following maternal separation. Int J Dev Neurosci. 2018;65:21-8.

15. Molet J, Maras PM, Avishai-Eliner S, Baram TZ. Naturalistic rodent models of chronic early-life stress. Dev Psychobiol. 2014;56:1675-88.

16. Carr CP, Martins CMS, Stingel AM, Lemgruber VB, Juruena MF. The role of early life stress in adult psychiatric disorders: a systematic review according to childhood trauma subtypes. J Nerv Ment Dis. 2013;201:1007-20.

17. Chocyk A, Majcher-Maoelanka I, Dudys D, Przyborowska A, Wêdzony K. Impact of early-life stress on the medial prefrontal cortex functions-a search for the pathomechanisms of anxiety and mood disorders. Pharm Rep. 2013;65:1462-70.

18. Reincke SAJ, Hanganu-Opatz IL. Early-life stress impairs recognition memory and perturbs the functional maturation of prefrontal-hippocampal-perirhinal networks. Sci Rep. 2017;7: 42042.

19. Holland FH, Ganguly P, Potter DN, Chartoff EH, Brenhouse HC. Early life stress disrupts social behavior and prefrontal cortex parvalbumin interneurons at an earlier time-point in females than in males. Neurosci Lett. 2014;566:131-6.

20. Yang Y, Cheng Z, Tang H, Jiao H, Sun X, Cui Q, et al. Neonatal maternal separation impairs prefrontal cortical myelination and cognitive functions in rats through activation of Wnt signaling. Cereb Cortex. 2017;27:2871-84. 
21. Mount CW, Monje M. Wrapped to adapt: experience-dependent myelination. Neuron. 2017;95:743-56.

22. Armbruster BN, Li X, Pausch MH, Herlitze S, Roth BL. Evolving the lock to fit the key to create a family of $G$ protein-coupled receptors potently activated by an inert ligand. Proc Natl Acad Sci USA. 2007;104:5163-8.

23. Anders S, Pyl PT, Huber W. HTSeq-a Python framework to work with high-throughput sequencing data. Bioinformatics. 2015;31:166-9.

24. Risso D, Ngai J, Speed TP, Dudoit S. Normalization of RNA-seq data using factor analysis of control genes or samples. Nat Biotechnol. 2014;32:896-902.

25. Robinson MD, McCarthy DJ, Smyth GK. edgeR: a Bioconductor package for differential expression analysis of digital gene expression data. Bioinformatics. 2010;26:139-40.

26. Storey JD, Tibshirani R. Statistical significance for genomewide studies. Proc Natl Acad Sci. 2003;100:9440-5.

27. Deacon RMJ. Digging and marble burying in mice: simple methods for in vivo identification of biological impacts. Nat Protoc. 2006;1:122-4.

28. Barker GRI, Bird F, Alexander V, Warburton EC. Recognition memory for objects, place, and temporal order: a disconnection analysis of the role of the medial prefrontal cortex and perirhinal cortex. J Neurosci. 2007;27:2948-57.

29. Hill RA, Patel KD, Goncalves CM, Grutzendler J, Nishiyama A. Modulation of oligodendrocyte generation during a critical temporal window after NG2 cell division. Nat Neurosci. 2014; $17: 1518-27$.

30. Silbereis JC, Huang EJ, Back SA, Rowitch DH. Towards improved animal models of neonatal white matter injury associated with cerebral palsy. Dis Model Mech. 2010;3:678-88.

31. Roth BL. DREADDs for neuroscientists. Neuron. 2016;89:683-94.

32. Stachniak TJ, Ghosh A, Sternson SM. Chemogenetic synaptic silencing of neural circuits localizes a hypothalamus $\rightarrow$ midbrain pathway for feeding behavior. Neuron. 2014;82:797-808.

33. David DJ, Samuels BA, Rainer Q, Wang J, Marsteller D, Mendez I, et al. Behavioral effects of fluoxetine in animal model od anxiety/depression are mediated by both neurogenesis-dependent and independent mechanisms. Neuron. 2010;62:479-93.

34. Makinodan M, Rosen KM, Ito S, Corfas G. A Critical period for social experience-dependent oligodendrocyte maturation and myelination. Sci (80-). 2012;337:1357-60.

35. Lehmann ML, Weigel TK, Elkahloun AG, Herkenham M. Chronic social defeat reduces myelination in the mouse medial prefrontal cortex. Sci Rep. 2017;7:46548.

36. Liu J, Dupree JL, Gacias M, Frawley R, Sikder T, Naik P, et al. Clemastine enhances myelination in the prefrontal cortex and rescues behavioral changes in socially isolated mice. J Neurosci. 2016;36:957-62.

37. Barrera K, Chu P, Abramowitz J, Steger R, Ramos RL, Brumberg JC. Organization of myelin in the mouse somatosensory barrel cortex and the effects of sensory deprivation. Dev Neurobiol. 2013;73:297-314.

38. Kikusui T, Kiyokawa Y, Mori Y. Deprivation of mother-pup interaction by early weaning alters myelin formation in male, but not female, ICR mice. Brain Res. 2007;1133:115-22.

39. Miki T, Yokoyama T, Kusaka T, Suzuki S, Ohta K, Warita K, et al. Early postnatal repeated maternal deprivation causes a transient increase in OMpg and BDNF in rat cerebellum suggesting precocious myelination. J Neurol Sci. 2014;336:62-7.

40. Bath KG, Manzano-Nieves G, Goodwill H. Early life stress accelerates behavioral and neural maturation of the hippocampus in male mice. Horm Behav. 2016;82:64-71.

41. Kikusui T, Mori Y. Behavioural and neurochemical consequences of early weaning in rodents. J Neuroendocrinol. 2009;21:427-31.
42. Minatohara K, Akiyoshi M, Okuno H. Role of immediate-early genes in synaptic plasticity and neuronal ensembles underlying the memory trace. Front Mol Neurosci. 2016;8:78.

43. Gibson EM, Purger D, Mount CW, Goldstein AK, Lin GL, Wood LS, et al. Neuronal activity promotes oligodendrogenesis and adaptive myelination in the mammalian brain. Sci (80-). 2014; 344:1252304-4.

44. Xiao L, Ohayon D, McKenzie IA, Sinclair-Wilson A, Wright JL, Fudge AD, et al. Rapid production of new oligodendrocytes is required in the earliest stages of motor-skill learning. Nat Neurosci. 2016;19:1210-7.

45. Covington HE, Lobo MK, Maze I, Vialou V, Hyman JM, Zaman $\mathrm{S}$, et al. Antidepressant effect of optogenetic stimulation of the medial prefrontal cortex. J Neurosci. 2010;30:16082-90.

46. He M, McCarthy KD. Oligodendroglial signal transduction systems are developmentally regulated. J Neurochem. 1994; 63:501-8.

47. Wright J, Zhang G, Yu T-S, Kernie SG. Age-related changes in the oligodendrocyte progenitor pool influence brain remodeling after injury. Dev Neurosci. 2010;32:499-509.

48. Orduz D, Maldonado PP, Balia M, Vélez-Fort M, de Sars V, Yanagawa $Y$, et al. Interneurons and oligodendrocyte progenitors form a structured synaptic network in the developing neocortex. Elife. 2015;4. https://doi.org/10.7554/eLife.06953.

49. Suri D, Teixeira CM, Cagliostro MKC, Mahadevia D, Ansorge MS. Monoamine-sensitive developmental periods impacting adult emotional and cognitive behaviors. Neuropsychopharmacology. 2014;40:88-112.

50. Takesian AE, Hensch TK. Changing brains - applying brain plasticity to advance and recover human ability. Merzenich M, Nahum M, Van Vleet T, editors Progress in brain research. vol. 207. Netherlands and UK: Elsevier; 2013. p. 3-34.

51. Gaspar P, Cases O, Maroteaux L. The developmental role of serotonin: news from mouse molecular genetics. Nat Rev Neurosci. 2003;4:1002-12.

52. Sarro EC, Wilson DA, Sullivan RM. Maternal regulation of infant brain state. Curr Biol. 2014;24:1664-9.

53. Bitzenhofer SH, Ahlbeck J, Wolff A, Wiegert JS, Gee CE, Oertner TG, et al. Layer-specific optogenetic activation of pyramidal neurons causes beta-gamma entrainment of neonatal networks. Nat Commun. 2017;8:14563.

54. Soiza-Reilly M, Meye FJ, Olusakin J, Telley L, Petit E, Chen X, et al. SSRIs target prefrontal to raphe circuits during development modulating synaptic connectivity and emotional behavior. Mol Psychiatry. 2019;24:726-45.

55. Gabbott PLa, Warner Ta, Jays PRL, Salway P, Busby SJ. Prefrontal cortex in the rat: Projections to subcortical autonomic, motor, and limbic centers. J Comp Neurol. 2005;492:145-77.

56. Fields RD. White matter in learning, cognition and psychiatric disorders. Trends Neurosci. 2008;31:361-70.

57. Schubert D, Martens GJM, Kolk SM. Molecular underpinnings of prefrontal cortex development in rodents provide insights into the etiology of neurodevelopmental disorders. Mol Psychiatry. 2015; 20:795-809.

58. Puig MV, Gulledge AT. Serotonin and prefrontal cortex function: neurons, networks, and circuits. Mol Neurobiol. 2011;44:1-16.

59. Carr CP, Martins CMS, Stingel AM, Lemgruber VB, Juruena MF. The role of early life stress in adult psychiatric disorders. J Nerv Ment Dis. 2013;201:1007-20.

60. Heim $C$, Nemeroff $C B$. The role of childhood trauma in the neurobiology of mood and anxiety disorders: preclinical and clinical studies. Biol Psychiatry. 2001;49:1023-39.

61. Edgar NM, Touma C, Palme R, Sibille E. Resilient emotionality and molecular compensation in mice lacking the oligodendrocytespecific gene Cnp1. Trans1 Psychiatry. 2011;1:e42-2. 
62. Chen X, Zhang W, Li T, Guo Y, Tian Y, Wang F, et al. Impairment of oligodendroglia maturation leads to aberrantly increased cortical glutamate and anxiety-like behaviors in juvenile mice. Front Cell Neurosci. 2015;9:467.

63. Dries DR, Zhu Y, Brooks MM, Forero DA, Adachi M, Cenik B, et al. Loss of nicastrin from oligodendrocytes results in hypomyelination and schizophrenia with compulsive behavior. J Biol Chem. 2016;291:11647-56.
64. Gould EA, Busquet N, Shepherd D, Dietz RM, Herson PS, Simoes de Souza FM, et al. Mild myelin disruption elicits early alteration in behavior and proliferation in the subventricular zone. Elife. 2018;7:e34783.

65. Birey F, Kloc M, Chavali M, Hussein I, Wilson M, Christoffel DJ, et al. Genetic and stress-induced loss of NG2 glia triggers emergence of depressive-like behaviors through reduced secretion of FGF2. Neuron. 2015;88:941-56. 